\title{
Structural analysis of urinary light chains and proteomic analysis of hyaline tubular casts in light chain associated kidney disorders
}

\author{
Thomas Reiter ${ }^{1}$, Daniela Knafl ${ }^{1}$, Hermine Agis ${ }^{2}$, Karl Mechtler $^{3}$, Ludwig Wagner $^{\text {Corresp., } 1}{ }^{\text {, Wolfgang Winnicki }}{ }^{1}$ \\ 1 Department of Medicine III, Division of Nephrology and Dialysis, Medical University of Vienna, Vienna, Austria \\ 2 Department of Medicine I, Division of Oncology, Medical University of Vienna, Vienna, Austria \\ 3 ProtChem Facility, IMP-IMBA, Research Institute of Molecular Pathology, Vienna, Austria \\ Corresponding Author: Ludwig Wagner \\ Email address: ludwig.wagner@meduniwien.ac.at
}

Background: Monoclonal overproduction of kappa and/or lambda light chains might result in renal light chain deposition disease. Light chain associated cast nephropathy and renal AL-amyloidosis represent two further pathologies going along with monoclonal gammopathy of renal significance and multiple myeloma. While cast nephropathy often manifests with acute kidney injury, AL-amyloidosis is rather accompanied with chronic kidney disease.

Methods: Urine samples were collected from 17 patients with multiple myeloma or monoclonal gammopathy. The urine sediment was stained for cast morphology by $\mathrm{H} / \mathrm{E}$ and light chain immunofluorescence. Following micro-selection of casts under microscope, proteomic analysis of casts was performed by mass spectrometry. Sucrose gradient sedimentation was employed and light chain architecture examined by immunoblotting. Uromodulin was measured by ELISA in sucrose gradient fractions.

Results: Urinary casts were observed of about $30 \mu \mathrm{m}$ in diameter by $\mathrm{H} / \mathrm{E}$ staining and under immunofluorescence microscopy. Casts with a diameter of $20 \mu \mathrm{m}$ were observed as a novel variant. Proteome analysis showed that in addition to the expected light chain variants produced by the malignant clone of plasma cells, also histones such as H2B and cathepsin B were contained. Uromodulin was not detectable in urinary casts of all patients. All eleven patients with lambda light chains showed predominant dimerized light chains in the urine immunoblot. Six patients with kappa light chains presented with predominantly monomeric forms of light chains in the immunoblot. The densitometric evaluated ratio of lambda dimers vs. monomers was significantly higher $(2.12 \pm 0.75)$ when compared with the ratio of kappa dimers vs. monomers $(0.64 \pm 0.47), p=0.00001$. Aggregates of light chains separated in part into denser sucrose fractions.

Conclusion: This work on urinary casts and light chains demonstrates that hyaline tubular casts represent a complex formation of protein-protein aggregates with histones and cathepsin B identified as novel cast components. Apart from the proteomic composition of the casts, also the formation of the light chains and aggregates is of relevance. Dimerized light chains, which are typical for lambda paraproteins, might be less dialyzable than monomeric forms and may therefore identify patients less responsive to high cut-off dialysis. 
2 Structural analysis of urinary light chains and proteomic analysis of hyaline tubular casts in light chain associated kidney disorders

4

5 Thomas Reiter ${ }^{1}$, Daniela Knafl ${ }^{1}$, Hermine Agis ${ }^{2}$, Karl Mechtler ${ }^{3}$, Ludwig Wagner ${ }^{1}$, Wolfgang 6 Winnicki $^{1}$

7

$8{ }^{1}$ Department of Medicine III, Division of Nephrology and Dialysis, Medical University of

9 Vienna, Austria

$10{ }^{2}$ Department of Medicine I, Division of Oncology, Medical University of Vienna, Austria

$11{ }^{3}$ ProtChem Facility, IMP-IMBA, Research Institute of Molecular Pathology, Vienna, Austria.

14 Corresponding Author:

15 Ludwig Wagner,

16 Waehringer Guertel 18-20, 1090 Vienna, Austria

17 Email adress: ludwig.wagner@meduniwien.ac.at 
21 Abstract

22 Background: Monoclonal overproduction of kappa and/or lambda light chains might result in

renal light chain deposition disease. Light chain associated cast nephropathy and renal ALamyloidosis represent two further pathologies going along with monoclonal gammopathy of renal significance and multiple myeloma. While cast nephropathy often manifests with acute kidney injury, AL-amyloidosis is rather accompanied with chronic kidney disease.

Methods: Urine samples were collected from 17 patients with multiple myeloma or monoclonal gammopathy. The urine sediment was stained for cast morphology by $\mathrm{H} / \mathrm{E}$ and light chain immunofluorescence. Following micro-selection of casts under microscope, proteomic analysis of casts was performed by mass spectrometry. Sucrose gradient sedimentation was employed and light chain architecture examined by immunoblotting. Uromodulin was measured by ELISA in sucrose gradient fractions.

Results: Urinary casts were observed of about $30 \mu \mathrm{m}$ in diameter by $\mathrm{H} / \mathrm{E}$ staining and under immunofluorescence microscopy. Casts with a diameter of $20 \mu \mathrm{m}$ were observed as a novel variant. Proteome analysis showed that in addition to the expected light chain variants produced by the malignant clone of plasma cells, also histones such as $\mathrm{H} 2 \mathrm{~B}$ and cathepsin $\mathrm{B}$ were contained. Uromodulin was not detectable in urinary casts of all patients.

All eleven patients with lambda light chains showed predominant dimerized light chains in the urine immunoblot. Six patients with kappa light chains presented with predominantly monomeric forms of light chains in the immunoblot. The densitometric evaluated ratio of lambda dimers vs. monomers was significantly higher $(2.12 \pm 0.75)$ when compared with the ratio of kappa dimers vs. monomers $(0.64 \pm 0.47), p=0.00001$. Aggregates of light chains separated in part into denser sucrose fractions. 
44 Conclusion: This work on urinary casts and light chains demonstrates that hyaline tubular casts

45 represent a complex formation of protein-protein aggregates with histones and cathepsin B

46 identified as novel cast components. Apart from the proteomic composition of the casts, also the

47 formation of the light chains and aggregates is of relevance. Dimerized light chains, which are

48 typical for lambda paraproteins, might be less dialyzable than monomeric forms and may

49 therefore identify patients less responsive to high cut-off dialysis. 
51

52

53

54

55

56

57

\section{Introduction}

Renal manifestation of multiple myeloma or elevated free light chains due to monoclonal gammopathy frequently manifest in light chain associated kidney disorders with different forms of deposits in renal compartments (Leung et al. 2019). The spectrum of renal complications associated with paraproteins is heterogeneous, with two major categories corresponding to the properties of the underlying B-cell clone. The first group is always associated with a high tumor mass and in this context cast nephropathy is of particular significance. The second group includes all patients with renal symptoms associated with a nephrotoxic monoclonal immunoglobulin as clonal proliferative disorder and is defined as monoclonal gammopathy of renal significance (MGRS) (Leung et al. 2012). Depending on results of immunofluorescence and electron microscopy, MGRS-associated diseases may include immunoglobulin light chain (AL) amyloidosis, monoclonal fibrillary glomerulonephritis (GN), immunotactoid GN, cryoglobulinaemic GN, light-chain proximal tubulopathy, crystal storing histiocytosis, monoclonal immunoglobulin deposition disease as well as proliferative glomerulonephritis and monoclonal immunoglobulin deposits (Leung et al. 2019; Leung et al. 2012).

Monoclonal overproduction of light chains by plasma cells represents the origin of the renal pathology. Since the publication of these studies nephrologists and oncologists have learned much in this direction (Hutchison et al. 2011; Manohar et al. 2018). Diagnostic procedures have been extended to include measurement of free light chain ratio in the serum, protein electrophoresis in serum and urine, immunofixation in serum and urine, bone marrow and renal biopsy (Leung 2016), whereas free light chain measurement in the urine is not recommended (Bradwell et al. 2003; Dispenzieri et al. 2009; Leung et al. 2019). Urine sediment testing for 
73 presence of urinary casts is simple, noninvasive and not harmful to patients and is included in

74 any diagnostic workup for patients with acute kidney injury (AKI).

75 Tubular cast formation has been attributed to specific non-covalent binding of light chains with

76 uromodulin proteins (Huang \& Sanders 1997). This is provoked when the proximal tubular

77 epithelia are overloaded with high concentrations of kappa or lambda light chains which under

78 normal conditions are reabsorbed by proximal tubular epithelial cells through a multiligand

79 endocytic receptor complex (Ying et al. 2012). This receptor complex is assumed to be

80

constituted out of megalin (Klassen et al. 2005) and cubilin (Batuman et al. 1998) as silencing of both genes resulted in ablation of free light chain induced uptake and toxicity in human renal proximal tubule epithelial cells (Ying et al. 2012). The primary structure of the monoclonal free light chain which results in a defined isoelectric point of the protein has much influence on the morphology and location of the light chain disease (Abraham et al. 2003) such as cast nephropathy (Cogne et al. 1991; Stevens 2000), AL-amyloidosis (Enqvist et al. 2007; Omtvedt et al. 2000; Stevens 2000) or monoclonal immunoglobulin deposition disease (del Pozo Yauner et al. 2008; Deret et al. 1997). However, individual patient-specific predisposing factors and medication (Holland et al. 1985; Ying \& Sanders 1998) might represent similar important players in renal disease morphology and progression. In this respect, secretion and reabsorption of other proteins and pharmacotherapeutic compounds (Luque et al. 2017) in urine appear to have a significant effect on cast formation. There is currently evidence that the human urinary proteome consists of more than 1000 individual protein fragments shaded or secreted into the urine (Liu et al. 2012; Marimuthu et al. 2011). It has already been reported that renal casts have variable hematoxylin/eosin staining intensities and might contain further compounds beyond uromodulin and light chain (Luque et al. 2017). 
96 These previous studies have motivated us to investigate the protein constituents of nephron

97 obstructing tubular casts through specific staining and micro-selection of these casts in the

98 urinary sediment. Casts are usually observed in the distal tubule, however, there is evidence that

99 they are also found in the proximal tubule and even in glomeruli (Start et al. 1988). In this study

100 analyzing urinary secreted casts, there are indications that thin casts might even originate from

101 the loop of Henle. Proteome analysis of these casts might help to shed further light on genesis

102 and potential treatment strategies in the clinical work up of patients with deranged free light

103 chain composition in urine. Free light chain dimerization or multimerization may influence

104 clearance properties when high cut-off membrane dialysis is intended. Therefore, in addition to

105 staining of urine sediment and proteomic analysis of casts, immunoblotting of urinary light

106 chains was an important focus of this study. 
107 Materials \& Methods

108 Urine sediment

109 Morning urine samples were collected from 17 patients with multiple myeloma or monoclonal

110 gammopathy of renal/undetermined significance. Demographics, clinical course of the patients

111 and their predominant paraprotein are depicted in Table 1. After centrifugation of the urine at

$1123000 \mathrm{~g}$ for 10 minutes, the supernatant was frozen at $-80^{\circ} \mathrm{C}$ for later use in fluid analysis. The

113 pellet was re-suspended in $2.5 \mathrm{~mL}$ cell culture media (RPMI 1640 containing 10\% calf serum)

114 and $100 \mu \mathrm{L}$ were applied to the funnel of a Shandon cyto-centrifuge. Following a spin at 1200

115 RPM for 3 minutes cytoslides were air dried for at least two hours and then either immediately

116 processed as described below or wrapped in aluminum foil and frozen at $-25^{\circ} \mathrm{C}$ for further

117 analysis.

118

119 Hematoxylin/Eosin staining was carried out as used in routine hematology staining of blood

120 smears. Slides were covered in Depex mounting media and a cover slip was applied for

121 microscopic observation.

122

123

Immunofluorescence for anti kappa/lambda staining (confocal microscopy) was carried out

after fixation in acetone for 4 minutes. The cell and cast containing area was surrounded by a fat pen (Dako) and the staining area was rewetted using PBS. For kappa/lambda staining goat (diluted 1:10000, A0191 and A0193 from Dako) or rabbit anti human kappa/lambda polyclonal antibody (Ab) (P0212, Dako) was applied (1:5000 in PBS) and incubated for two hours at room temperature following two washes in PBS each for 10 minutes, the donkey anti goat or goat anti rabbit (Alexa 488, dilution 1:1000 in PBS) was applied for 1 hour at room temperature. Before 
130 washing the slide $20 \mu \mathrm{L}$ of DAPI solution was spotted onto the Ab containing area. Following

131 the final wash Vetashield mounting media and cover slip were applied and the slides were

132 recorded under a Zeiss invert confocal microscope using ZEN program for picture recording.

133

134 Urinary cast isolation

135 Urine sediment generated as described above was air dried from patients presenting with AL-

136 amyloidosis and cast nephropathy or presenting with cast nephropathy alone. Cytopreparations

137 were viewed under phase contrast light microscope (Labovert FS, Leitz, Germany) and

138 individual casts were picked using the tip of specifically gas flame pulled glass Pasteur pipettes

139 under microscopic observation. Five casts were placed into a $400 \mu \mathrm{L}$ PCR tube containing

$14010 \mathrm{~b} \mu \mathrm{L}$ PBS. This represented the work up starting material for proteome analysis.

142 Substructural fractionation on a sucrose gradient

143 Cells and cell nuclei and large aggregates were pelleted by centrifugation at 1500 RPM. Five

144 hundred $\mu \mathrm{L}$ of resultant supernatant containing cast fragments and micro-aggregates and

145 exosomes were loaded on to a discontinuous sucrose gradient in Ultra-clear centrifuge tubes

146 (Beckman, 344062) at $4^{\circ} \mathrm{C}$. Following two hours centrifugation at 40000 RPM using an SW60

147 rotor in an L-80 ultracentrifuge the gradient was immediately fractionated in $200 \mu \mathrm{L}$ aliquots

148 using a peristaltic pump starting at the bottom of the tube. Individual fractions were subjected to

149 immunoblotting using antibodies for kappa and lambda light chains (Dako as above) and 150 aquaporin 1 (AQP1, Millipore).

151

152 Immunoblotting 
153 Twenty $\mu \mathrm{L}$ of individual fractions of urine were loaded onto a 12\% SDS PAGE gel and run

154 under denaturing conditions. Following the transfer onto nitrocellulose the proteins were stained

155 using kappa and lambda specific antibodies (Dako) as well as Ab against AQP1 (Millipore). The

156 specific protein staining was developed by HRP labeled goat anti rabbit affinity purified

157 antibodies (Dako). After each staining procedure the blot was washed using TPBS for 10

158 minutes twice. Chemiluminescence reagent was applied to the blot which was visualized using

159 Fusion software at the luminescence recorder (Fusion Fx, Vilber Lourmat). Pictures were further

160 processed using Photoshop version 6. Densitometric evaluation of individual signals of dimers

161 vs. monomers were performed using the Fusion software (Fusion Fx, Vilber Lourmat).

\section{Uromodulin ELISA test}

164 Uromodulin concentration was measured in the same sucrose density gradient fractions as used

165 for immunoblotting by a commercially available ELISA assay (BioVendor, Czech Republic).

166 The Elisa was performed as suggested in the company's test manual. In brief, fractions were

167 diluted in sample dilution buffer and incubated together with the standard series. Following the 168 incubation with biotinylated detection antibody and streptavidin-HRP conjugate the signal was

169 developed with TMB substrate and read by an ELISA reader. Concentrations were calculated 170 according the standard curve. The detection range of the ELISA assay is 0.5 to $25 \mathrm{ng} / \mathrm{mL}$.

\section{Mass spectrometry}

173 NanoLC-MS Analysis: Trypsin digestion was carried out in solution and resultant peptides were

174 purified on a C18 column before injection into the UltiMate 3000 RSLC nano HPLC system 175 (Thermo Fisher Scientific, Amsterdam, Netherlands). This was connected to a Q Exactive HF mass 
176 spectrometer (Thermo Fisher Scientific, Bremen, Germany) with a Proxeon nanospray source

177 (Thermo Fisher Scientific, Odense, Denmark). Initial loading was carried out onto a trap column

178 (Thermo Fisher Scientific, Amsterdam, Netherlands, PepMap C18, $5 \mathrm{~mm} \times 300 \mu \mathrm{m}$ ID, $5 \mu \mathrm{m}$

179 particles, $100 \AA$ pore size) at a flow rate of $25 \mu \mathrm{L}$ min- 1 using $0.1 \%$ TFA as mobile phase. This

180 column was moved in line with the analytical column (Thermo Fisher Scientific, Amsterdam,

181 Netherlands, PepMap C18, $500 \mathrm{~mm} \times 75 \mu \mathrm{m}$ ID, $2 \mu \mathrm{m}, 100 \AA$ ) following a 10 minute flow.

182 Peptides were eluted by a binary $1 \mathrm{~h}$ gradient with a flow rate of $230 \mathrm{~nL}$ min- 1 . The applied gradient

183 for starting represented: 98\% A (water/formic acid, 99.9/0.1, v/v) and 2\% B

184 (water/acetonitrile/formic acid, 19.92/80/0.08, v/v/v). Over a period of 60 minutes B was increased

185 to $35 \%$ and over the next 5 minutes B was further increased up to $90 \%$ and was kept in a plateau

186 for 5 minutes. Within the next 2 minutes the gradient was returned back to $98 \% \mathrm{~A}$ and $2 \% \mathrm{~B}$. This

187 was the setting for equilibration at $30^{\circ} \mathrm{C}$.

188 The mass spectrometer was set to data-dependent mode in full scan ( $\mathrm{m} / \mathrm{z}$ range $380-1650$, nominal 189 resolution of 120,000 , target value 3E6) followed by MS/MS scans of the 10 most abundant ions. $190 \mathrm{MS} / \mathrm{MS}$ spectra were obtained using normalized collision energy of $27 \%$, isolation width of $2 \mathrm{~m} / \mathrm{z}$, 191 resolution of 30,000 and the target value was set to 1E5. Precursor ions selected for fragmentation 192 were read at dynamic exclusion list for $20 \mathrm{sec}$. Additionally, the minimum AGC target was set to 193 2E4. The intensity threshold was calculated to be 8E4.

\section{Data Processing protocol and peptide identification:}

196 The Proteome Discoverer (version 2.3.0.523, Thermo Scientific) was fed with RAW-data files.

197 The generated MS/MS spectra have been searched using MSAmanda v2.0.0.9849 (Dorfer et al. 198 2014). RAW-files were searched against the SwissProt human database $(20,169$ sequences; 
19911315,794 residues). The search parameters were: peptide mass tolerance \pm 5 ppm as well as the

200 fragment mass tolerance was limited to 0.03 Da. Missed cutting sites were 2. The resultant output

201 was filtered to $1 \%$ false discovery rate using again Thermo Proteome Discoverer. Peptide area

202 under the curve was quantified using IMP-apQuant (Doblmann et al. 2019).

\section{Statistical analyses}

204 We present quotients as means \pm standard deviation (SD). Differences of lambda and kappa

205 dimer vs. monomer ratio were analyzed with paired t-test. Data management and analysis was

206 conducted by Microsoft Excel (C) Microsoft, Redmont, WA). The statistical testing performed

207 was two-sided and a p-value $\leq 0.05$ was considered significant.

208

209 Ethics approval and consent to participate

210 This study involves human participants. Urine samples were obtained from adult study

211 participants and/or their legal guardians older than 18 years who have given written informed

212 consent. All methods of experiments involving human participants were carried out according to

213 relevant guidelines. All experimental protocols involving human participants received approval

214 of the Ethics Committee of the Medical University of Vienna (EK 1043/2016).

215

216

217

218

219

Peer) reviewing PDF | (2019:06:38914:1:1:NEW 22 Aug 2019) 


\section{Results}

\section{Cast morphology and constituent analysis}

222 Hematoxylin/Eosin staining of tubular casts revealed various staining intensities as well as

223 colour variation. Colour variation results from different contents, which either are more

224 basophilic or acidophilic depending on constituents. As demonstrated in Figure 1A,C,D three

225 casts are more eosinophilic when compared to one in Figure 1B that shows a fainter staining.

226 The thinner cast at Figure 1C is originating from a different stretch of the nephron than the other

227 cast fragments, such as the the loop of Henle. Three tubular cells are incorporated in this thin

228 cast.

229 In order to further study the constituents of tubular casts we sought for the incorporation of light

230 chain components. As already reported in earlier work tubular casts contain the light chain (LC)

231 paraprotein such as also measured in serum. As shown in Figure 2A and 2B the lambda light

232 chain is incorporated at the tubular cast which has a thinner diameter at the left side upward

233 directed end when compared with the other part. This picture further confirms the assumption

234 that casts can originate from thinner stretches of the nephron.

235 Analysis of casts by electron microscopy has identified various fine structures in previous studies

236 (Uribe-Uribe \& Herrera 2006) and the most dominant variant was represented by the hyaline

237 form, however, the biochemical proteinaceous constituents of hyaline casts in light chain

238 associated kidney disorders has not been the subject of investigation before. Only uromodulin

239 was documented as light chain interaction partner. Therefore we sought by mass spectrometry to

240 analyze the proteome of isolated urinary casts from patient 14 who had no detectable uromodulin

241 in the sucrose density fractionation. In these experiments we identified the patient's lambda LC

242 and histones in particular H2B sub-variants (Table 2) as a dominant additional constituents of 
243 the cast proteome. It is of interest that a urinary protease such as cathepsin B was also contained

244 in this complex (Table 2). In repeated proteome analyses (n=3) H2 variants were similarly

245 highly present. Depending on the cast type chosen for analysis more or less cellular proteins

246 were recovered. However, this study was focused on hyaline casts. Of particular note is the

247 observation that as shown in (Supplementary Figure 1) patient 14 had received

248 immunomodulatory therapy 2.5 days before the onset of AKI stage 3 according to KDIGO. His

249 urine sediment was filled with hyaline casts. The liberation of histones from apoptotic plasma

250 cells and the filtration into the urine might have represented the main provocation of cast

251 formation in the renal tubules as uromodulin was not detectable. His lambda light chain analysis

252 in the density gradient fractions (Figure 3A) showed to some extent presence of aggregated LC

253 signals in density fractions 6-10 representing most likely complexes but there was almost no

254 presence of LCs in exosome containing fractions 2-6. Presence of exosomes was verified by

255 immunoblotting of the same fractions with Ab against AQP1 a tubular cell membrane protein

256 (Figure 3B). By a second approach using urine SDS PAGE analysis and immunoblotting lambda

257 LCs appeared predominantly as dimers in urine and made up 78\% in the densitometric analysis

258 (Figure 4, lane 14). No amyloid deposition had been observed in the kidney biopsy of this

259 patient although the duration of disease had been lasting for already 5 years (Table 1). The

260 patient underwent high cut-off dialysis and as demonstrated in Supplementary Figure 1 his

261 renal function improved.

262

263 Analysis of urinary light chain structure

264 As already documented in earlier work not the concentration but rather the structure and 265 morphology represents the underlying cause for manifestation of renal light chain disease. For 
266 this reason we analyzed the light chain dimerization in 17 myeloma or monoclonal gammopathy

267 patients (Table 1). As demonstrated in Figure 4 patients positive for lambda LCs showed

268 patterns with higher quantities of LC dimers than monomers. Furthermore, eight patients out of

26911 positive for lambda LC developed CKD stages 4-5 according to KDIGO (Table 1). Patients

$2703,8,9,10,13$ and 14 were highly positive for lambda dimers in the immunoblots (Figure 4).

271 Dimerization of lambda LC could be entirely resolved into monomers by adding DDT as

272 reducing agent in the sample buffer (Figure 4B).

273 Patients positive for kappa LCs showed patterns with higher quantities of LC monomers than

274 dimers (Figure 5). The densitometric analysis evaluated ratio of lambda dimers vs. monomers

275 was significantly higher $(2.12 \pm 0.75)$ when compared with the ratio of kappa dimers vs.

276 monomers $(0.64 \pm 0.47), \mathrm{p}=0.00001$.

277 We further intended to elaborate the light chain aggregates or complex formation resulting in

278 renal light chain disease by density gradient centrifugation in patients 13,14 and 15 . In the urine

279 sediment of amyloidosis patient 13 the light chain was found in both the soluble urine proteins

280 (Figure 6A, fractions 11-14) and to a lesser extent in the membrane fractions (Figure 6A, lanes

281 1-4). Membrane exosome fractions were identified by AQP1 blotting of the same fractions

282 (Figure 6B). This was similar for patient 15 despite the fact that his disease was linked to kappa

283 LC which was going along with AKI stage 3 (Figure 7A). Membrane fractions represented by

284 lanes 1-4 containing exosomes and cast particles were identified by presence of AQP1 (Figure

285 7B).

286 The Uromodulin protein (UMOD) has been shown earlier to represent the binding partner for

287 LCs in forming casts. In this study we did not find uromodulin protein in the casts of patient 14,

288 which was confirmed by absence in sucrose gradient density fractions and urine. In contrast, 
289 there was a small peak concentration in heavy membrane fraction 2 in patients 13 and 15 (Figure 290 6C and Figure 7C). This fractions corresponded with presence of exosomes and cast fragments 291 in the gradient sedimentation experiments. Fractions 11-14 correspond to soluble molecules in 292 urine. In patients 13 and 15 urine uromodulin concentration was high (Figure 6C and Figure 293 7C). 


\section{Discussion}

295 This study investigates hyaline cast constituents and factors involved in cast formation as well as

296 urine light chain multimeric conformation. It documents that casts of thinner diameter than

297 assumed can be observed in light chain disease which might originate from the loop of Henle.

298 Selective cast harvesting by micromanipulation under microscope observation for proteome

299 analysis revealed histones as major constituents of casts together with the light chain present as

300 serum and urine paraprotein. Rather unexpectedly, cathepsin B was also found among the

301 dominant cast constituents. In addition, all patients with lambda light chains showed

302 dimerization of urine light chains in the immunoblot and were more likely to develop

303 amyloidosis and chronic kidney disease.

304 Secretion of light chains has been shown in previous studies to predominantly occur as

305 monomers in vitro (Dul et al. 1996) but this does not have to apply to multiple myeloma patients

306 in vivo. In our study patients positive for lambda LCs showed patterns with higher quantities of

307 LC dimers than monomers in urine immunoblotting. In contrast, patients positive for kappa LCs

308 showed patterns with higher quantities of LC monomers than dimers. This may represent an

309 important observation for potential clinical treatment options such as high cut-off dialysis

310 (Bridoux et al. 2017; Finkel 2014). The much smaller monomeric forms might represent better

311 candidates for clearance through dialysis, whereas dimers may require longer dialysis and may

312 be less cleared from plasma. It would therefore be reasonable to test patients for LC structure and

313 size morphology to assess the potential benefit of high cut-off hemodialysis.

314 Earlier work (Korbet \& Schwartz 2006; Luque et al. 2017) and this study confirm that cast

315 formation and amyloid fibrilogenesis (Kim et al. 2000) represent complex physicochemical and

316 biochemical processes (Radamaker et al. 2019). Interaction of electrostatic charges between 
317 proteins such as uromodulin (Huang \& Sanders 1997) together with nephron-specific biological

318 processes such as incorporation of exosomes shaded from tubular epithelial brush border are of

319 importance. In addition, proteins filtered through the glomerulum and not reabsorbed by the

320 proximal tubular epithelia seem to represent important players in hyaline cast formation.

321 In our cast experiments histones getting liberated from apoptotic cells during chemotherapy,

322 representing small positively charged molecules of $13-17 \mathrm{kDa}$, which are filtered through the

323 glomerular membrane, seem to have acted as partners for LCs to form hyaline casts. In this

324 situation no uromodulin concentrations in the urine could be measured of the patient having

325 undergone immunomodulatory therapy and AKI. Thus it has to be suggested, that cast formation

326 might be induced by various proteins and biological compounds (Luque et al. 2017) beyond

327 uromodulin. Hence, novel considerations in management of cast nephropathy might be important

328 (Manohar et al. 2018; Ying et al. 2012).

329 It has been documented earlier that Bence Jones protein and amyloid fibril generated out of LCs

330 are C-terminally cleaved (Enqvist et al. 2009; Terry et al. 1973). In this line the abundant

331 presence of Cathepsin B in hyaline casts represents a protease known for cleaving C-terminal

332 parts in the process of activation/inactivation of various biologically active compounds (Kumar

333 et al. 2018). It is therefore of interest that Cathepsin B might be involved in proteolytic

334 processing of the C-terminal part of the cast components in particular the LCs. Cathepsin B is

335 most likely involved in proteolytic digestion and thereby mobilization of casts from the site of

336 tubular obstruction. It is of note that in fraction 14 of Figure 7A (soluble urine proteins) the light

337 chain dimer is of reduced size probably because of cleavage, which must have been achieved by

338 urine contained proteases such as cathepsins. 
339 Brush border specific proteins are contained in the hyaline casts isolated from the urine sediment

340 in individual specimens. Some of the patient derived hyaline casts contained cells to various

341 extent. This demonstrates that cast formation at a specific stretch of the nephron causes injury to

342 tubular epithelial cells or cells subsequently loosen from the basal membrane when the tubule

343 dilates proximal to the part, which had been obstructed by the cast. Proteases incorporated (such

344 as cathepsin) might contribute to cast liberation because of proteolytic digestion and thereby

345 shrinkage of the cast.

346 We found kappa and lambda LCs in the heavy fractions of the sucrose gradient at densities

347 where exosomes and cast fragments were expected. This revealed that LCs are present in

348 microsomes where AQP1 is present or it represents cast fragments.

349 The molecular size of LC in serum must have an important impact on its clearance by the

350 glomerulum and by high cut-off dialysis membranes. LC testing using urine immunoblotting to

351 determine the molecular size of LC proteins appears to be an important option for evaluating

352 whether a patient could benefit from high cut-off dialysis treatment. Lambda-positive patients

353 known for the presence of higher polymers (Sallee \& Burtey 2019), as confirmed in this study,

354 may not be good candidates for LC clearance through high-cut off dialysis membranes.

355 The main limitation of this study is that no clinically available quantitative methods are available

356 for measurement of LC dimers versus monomers, therefore this study uses semi-quantitative

357 methods. Casts for proteomic analysis could not be isolated from all patients and not all patients

358 underwent renal biopsy. Due to the limits of proteomic databases, only well-known peptide

359 sequences can be detected by proteome analysis. In respect of clinical treatment regimens this

360 structural analysis of casts and light chains is preliminary and focused on a small sample size.

361 This may encourage clinical researchers at the same time to conduct studies in larger patient 
362 groups on different treatment regimens, in particular on lambda/kappa light-chain associated

363 kidney disorders. It is of note, that in our study for the first time proteomic analysis was

364 performed on micro-selected cast structures.

365

\section{Conclusions}

367 This study demonstrates that hyaline tubular casts represent a complex formation of protein368 protein aggregates with histones and cathepsin B identified as novel cast components. Apart

369 from the proteomic cast composition, the formation of light chains and aggregates is of particular 370 significance. Dimerized light chains typical for lambda paraproteins might be less dialyzable

371 than monomeric forms. Therefore, testing for light chain structure may select patients who are

372 more likely to benefit from dialysis using high cut-off membranes, but clinical trials are required 373 to confirm this. 


\section{References}

375 Abraham RS, Geyer SM, Price-Troska TL, Allmer C, Kyle RA, Gertz MA, and Fonseca R. 2003. Immunoglobulin light chain variable (V) region genes influence clinical presentation and outcome in light chain-associated amyloidosis (AL). Blood 101:38013808. 10.1182/blood-2002-09-2707

Batuman V, Verroust PJ, Navar GL, Kaysen JH, Goda FO, Campbell WC, Simon E, Pontillon F, Lyles M, Bruno J, and Hammond TG. 1998. Myeloma light chains are ligands for cubilin (gp280). The American journal of physiology 275:F246-254.

10.1152/ajprenal.1998.275.2.F246

Bradwell AR, Carr-Smith HD, Mead GP, Harvey TC, and Drayson MT. 2003. Serum test for assessment of patients with Bence Jones myeloma. Lancet 361:489-491. Doi $10.1016 / \mathrm{S} 0140-6736(03) 12457-9$

Bridoux F, Carron PL, Pegourie B, Alamartine E, Augeul-Meunier K, Karras A, Joly B, Peraldi MN, Arnulf B, Vigneau C, Lamy T, Wynckel A, Kolb B, Royer B, Rabot N, Benboubker L, Combe C, Jaccard A, Moulin B, Knebelmann B, Chevret S, and Fermand JP. 2017. Effect of High-Cutoff Hemodialysis vs Conventional Hemodialysis on Hemodialysis Independence Among Patients With Myeloma Cast Nephropathy: A Randomized Clinical Trial. JAMA 318:2099-2110. 10.1001/jama.2017.17924

Cogne M, Preud'homme JL, Bauwens M, Touchard G, and Aucouturier P. 1991. Structure of a monoclonal kappa chain of the V kappa IV subgroup in the kidney and plasma cells in light chain deposition disease. The Journal of clinical investigation 87:2186-2190. 10.1172/JCI115252 
396

397

398

399

400

401

402

403

404

405

406

407

408

409

410

411

412

413

414

415

416

417

del Pozo Yauner L, Ortiz E, Sanchez R, Sanchez-Lopez R, Guereca L, Murphy CL, Allen A, Wall JS, Fernandez-Velasco DA, Solomon A, and Becerril B. 2008. Influence of the germline sequence on the thermodynamic stability and fibrillogenicity of human lambda 6 light chains. Proteins 72:684-692. 10.1002/prot.21934

Deret S, Chomilier J, Huang DB, Preud'homme JL, Stevens FJ, and Aucouturier P. 1997. Molecular modeling of immunoglobulin light chains implicates hydrophobic residues in non-amyloid light chain deposition disease. Protein engineering 10:1191-1197.

Dispenzieri A, Kyle R, Merlini G, Miguel JS, Ludwig H, Hajek R, Palumbo A, Jagannath S, Blade J, Lonial S, Dimopoulos M, Comenzo R, Einsele H, Barlogie B, Anderson K, Gertz M, Harousseau JL, Attal M, Tosi P, Sonneveld P, Boccadoro M, Morgan G, Richardson P, Sezer O, Mateos MV, Cavo M, Joshua D, Turesson I, Chen W, Shimizu K, Powles R, Rajkumar SV, Durie BGM, and Grp IMW. 2009. International Myeloma Working Group guidelines for serum-free light chain analysis in multiple myeloma and related disorders. Leukemia 23:215-224. 10.1038/leu.2008.307

Doblmann J, Dusberger F, Imre R, Hudecz O, Stanek F, Mechtler K, and Durnberger G. 2019. apQuant: Accurate Label-Free Quantification by Quality Filtering. Journal of Proteome Research 18:535-541. 10.1021/acs.jproteome.8b00113

Dorfer V, Pichler P, Stranzl T, Stadlmann J, Taus T, Winkler S, and Mechtler K. 2014. MS Amanda, a universal identification algorithm optimized for high accuracy tandem mass spectra. Journal of proteome research 13:3679-3684. 10.1021/pr500202e

Dul JL, Aviel S, Melnick J, and Argon Y. 1996. Ig light chains are secreted predominantly as monomers. Journal of immunology 157:2969-2975. 
418 Enqvist S, Sletten K, Stevens FJ, Hellman U, and Westermark P. 2007. Germ line origin and

419

420

421

422

423

424

425

426

427

428

429

430

431

432

433

434

435

436

437

438

439 somatic mutations determine the target tissues in systemic AL-amyloidosis. PloS one 2:e981. 10.1371/journal.pone.0000981

Enqvist S, Sletten K, and Westermark P. 2009. Fibril protein fragmentation pattern in systemic AL-amyloidosis. The Journal of pathology 219:473-480. 10.1002/path.2607

Finkel KW. 2014. Is high cut-off hemodialysis effective in myeloma kidney? Seminars in dialysis 27:234-236. 10.1111/sdi.12210

Holland MD, Galla JH, Sanders PW, and Luke RG. 1985. Effect of urinary pH and diatrizoate on Bence Jones protein nephrotoxicity in the rat. Kidney international 27:46-50.

Huang ZQ, and Sanders PW. 1997. Localization of a single binding site for immunoglobulin light chains on human Tamm-Horsfall glycoprotein. The Journal of clinical investigation 99:732-736. 10.1172/JCI119218

Hutchison CA, Batuman V, Behrens J, Bridoux F, Sirac C, Dispenzieri A, Herrera GA, Lachmann H, and Sanders PW. 2011. The pathogenesis and diagnosis of acute kidney injury in multiple myeloma. Nature reviews Nephrology 8:43-51.

10.1038/nrneph.2011.168

Kim Y, Wall JS, Meyer J, Murphy C, Randolph TW, Manning MC, Solomon A, and Carpenter JF. 2000. Thermodynamic modulation of light chain amyloid fibril formation. The Journal of biological chemistry 275:1570-1574. 10.1074/jbc.275.3.1570

Klassen RB, Allen PL, Batuman V, Crenshaw K, and Hammond TG. 2005. Light chains are a ligand for megalin. Journal of applied physiology 98:257-263.

10.1152/japplphysiol.01090.2003

Peer) reviewing PDF | (2019:06:38914:1:1:NEW 22 Aug 2019) 
440 Korbet SM, and Schwartz MM. 2006. Multiple myeloma. Journal of the American Society of

441

442

443

444

445

446

447

448

449

450

451

452

453

454

455

456

457

458

459

460

461

Nephrology : JASN 17:2533-2545. 10.1681/ASN.2006020139

Kumar A, Daitsh Y, Ben-Aderet L, Qiq O, Elayyan J, Batshon G, Reich E, Maatuf YH, Engel S, and Dvir-Ginzberg M. 2018. A predicted unstructured C-terminal loop domain in SIRT1 is required for cathepsin B cleavage. Journal of cell science 131. 10.1242/jcs.214973

Leung N. 2016. To biopsy or not to biopsy, that is the question in myeloma cast nephropathy. Nephrology, dialysis, transplantation : official publication of the European Dialysis and Transplant Association - European Renal Association 31:1-3. 10.1093/ndt/gfv294

Leung N, Bridoux F, Batuman V, Chaidos A, Cockwell P, D'Agati VD, Dispenzieri A, Fervenza FC, Fermand JP, Gibbs S, Gillmore JD, Herrera GA, Jaccard A, Jevremovic D, Kastritis E, Kukreti V, Kyle RA, Lachmann HJ, Larsen CP, Ludwig H, Markowitz GS, Merlini G, Mollee P, Picken MM, Rajkumar VS, Royal V, Sanders PW, Sethi S, Venner CP, Voorhees PM, Wechalekar AD, Weiss BM, and Nasr SH. 2019. The evaluation of monoclonal gammopathy of renal significance: a consensus report of the International Kidney and Monoclonal Gammopathy Research Group. Nature Reviews Nephrology $15: 45-59.10 .1038 / \mathrm{s} 41581-018-0077-4$

Leung N, Bridoux F, Hutchison CA, Nasr SH, Cockwell P, Fermand JP, Dispenzieri A, Song KW, and Kyle RA. 2012. Monoclonal gammopathy of renal significance: when MGUS is no longer undetermined or insignificant. Blood 120:4292-4295. 10.1182/blood-2012-07445304

Liu X, Shao C, Wei L, Duan J, Wu S, Li X, Li M, and Sun W. 2012. An individual urinary proteome analysis in normal human beings to define the minimal sample number to 

70

464

465

466

467

468

469

470

471

472

473

474

475

476

477

478

479

480

481

482

483

Luque Y, Louis K, Jouanneau C, Placier S, Esteve E, Bazin D, Rondeau E, Letavernier E, Wolfromm A, Gosset C, Boueilh A, Burbach M, Frere P, Verpont MC, Vandermeersch S, Langui D, Daudon M, Frochot V, and Mesnard L. 2017. Vancomycin-Associated Cast Nephropathy. Journal of the American Society of Nephrology : JASN 28:1723-1728. 10.1681/ASN.2016080867

Manohar S, Nasr SH, and Leung N. 2018. Light Chain Cast Nephropathy: Practical Considerations in the Management of Myeloma Kidney-What We Know and What the Future May Hold. Current hematologic malignancy reports 13:220-226. 10.1007/s11899018-0451-0

Marimuthu A, O'Meally RN, Chaerkady R, Subbannayya Y, Nanjappa V, Kumar P, Kelkar DS, Pinto SM, Sharma R, Renuse S, Goel R, Christopher R, Delanghe B, Cole RN, Harsha HC, and Pandey A. 2011. A comprehensive map of the human urinary proteome. Journal of proteome research 10:2734-2743. 10.1021/pr2003038

Omtvedt LA, Bailey D, Renouf DV, Davies MJ, Paramonov NA, Haavik S, Husby G, Sletten K, and Hounsell EF. 2000. Glycosylation of immunoglobulin light chains associated with amyloidosis. Amyloid : the international journal of experimental and clinical investigation : the official journal of the International Society of Amyloidosis 7:227-244. $10.3109 / 13506120009146437$

Radamaker L, Lin YH, Annamalai K, Huhn S, Hegenbart U, Schonland SO, Fritz G, Schmidt M, and Fandrich M. 2019. Cryo-EM structure of a light chain-derived amyloid fibril from a 
484

485

486

487

488

489

490

491

492

493

494

495

496

497

498

499

500

501

502

503

504

505

patient with systemic AL amyloidosis. Nature communications 10:1103.

10.1038/s41467-019-09032-0

Sallee M, and Burtey S. 2019. Myeloma cast nephropathy: the dusk of high cutoff haemodialysis. The Lancet Haematology 6:e174-e176. 10.1016/S2352-3026(19)30044-4

Start DA, Silva FG, Davis LD, D'Agati V, and Pirani CL. 1988. Myeloma cast nephropathy: immunohistochemical and lectin studies. Modern pathology : an official journal of the United States and Canadian Academy of Pathology, Inc 1:336-347.

Stevens FJ. 2000. Four structural risk factors identify most fibril-forming kappa light chains. Amyloid : the international journal of experimental and clinical investigation : the official journal of the International Society of Amyloidosis 7:200-211.

Terry WD, Page DL, Kimura S, Isobe T, Osserman EF, and Glenner GG. 1973. Structural identity of Bence Jones and amyloid fibril proteins in a patient with plasma cell dyscrasia and amyloidosis. The Journal of clinical investigation 52:1276-1281. 10.1172/JCI107295

Uribe-Uribe NO, and Herrera GA. 2006. Ultrastructure of Tubular Casts. Ultrastructural Pathology 30:159-166. 10.1080/01913120600689749

Ying WZ, Allen CE, Curtis LM, Aaron KJ, and Sanders PW. 2012. Mechanism and prevention of acute kidney injury from cast nephropathy in a rodent model. The Journal of clinical investigation 122:1777-1785. 10.1172/JCI46490

Ying WZ, and Sanders PW. 1998. Dietary salt regulates expression of Tamm-Horsfall glycoprotein in rats. Kidney international 54:1150-1156. 10.1046/j.15231755.1998.00117.x 
Figure 1

$H / E$ stained casts on cyto-slide preparations obtained from a patient with lambda light chain myeloma.

Urine sediment obtained in the context of AKI stage 3 revealed multiple casts. The average cast diameter comprised about $30 \mu \mathrm{m}$ (A, B, D). Considerable thinner cast containing tubular cells most likely originating from a thinner part of the nephron (C). Pale staining tubular cast originating from the distal tubule (B). 


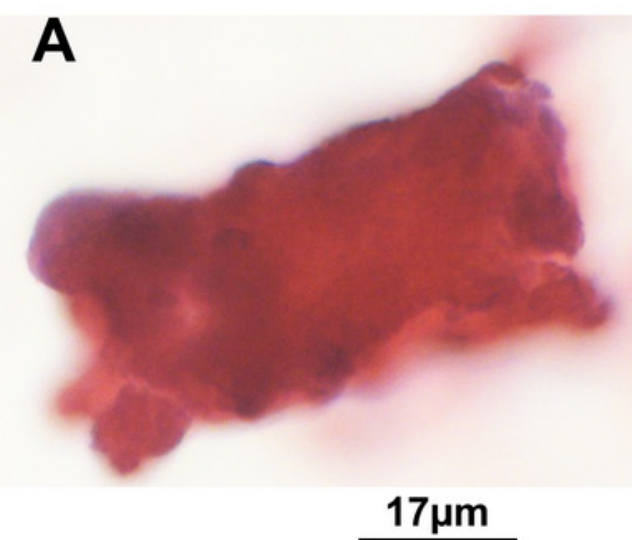

B
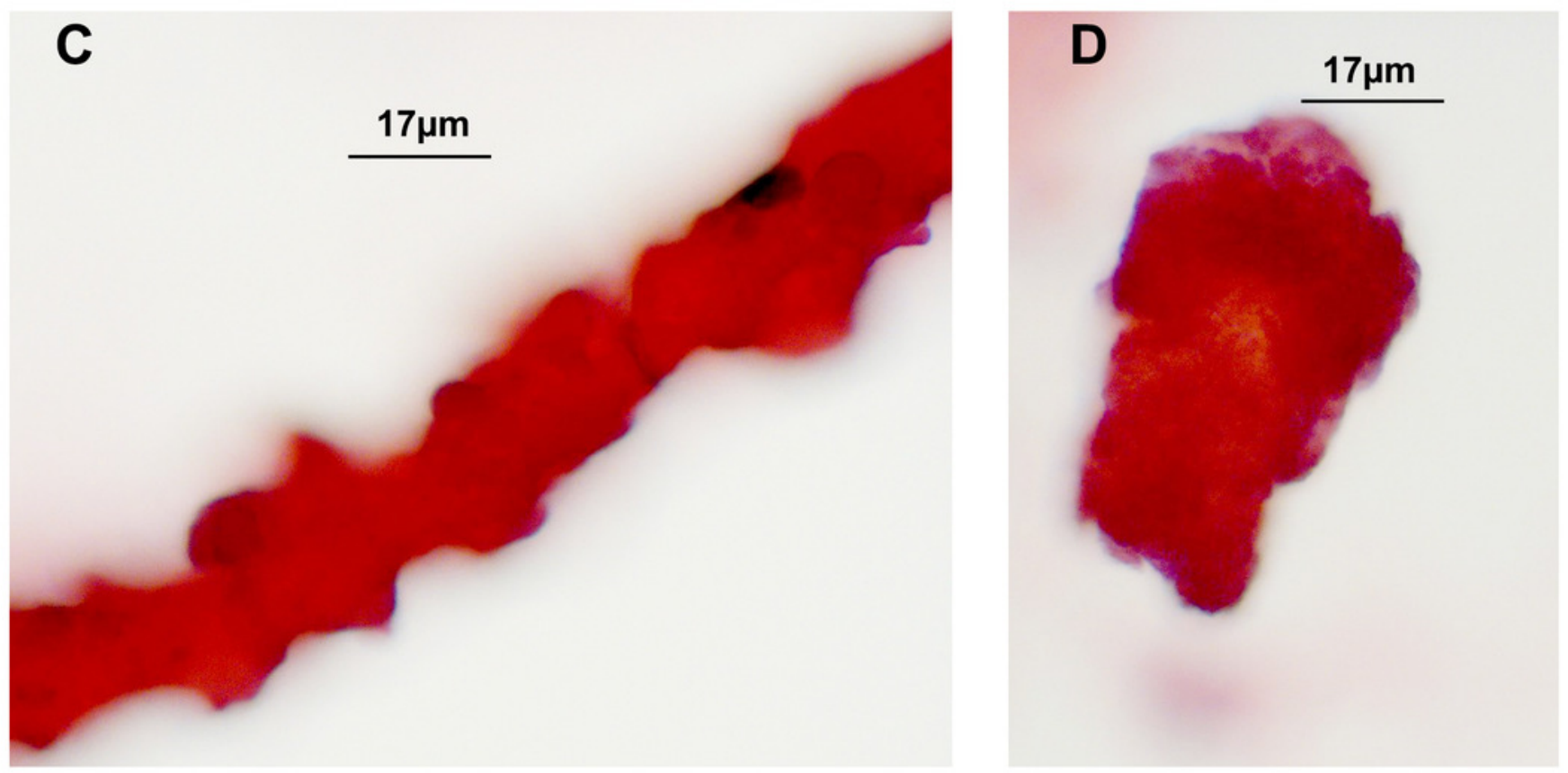


\section{Figure 2}

Immunofluorescence staining of renal tubular casts. Various staining intensity for lambda light chain of tubular casts.

The cast is bent and shows a conical structure with the thinner part directed upwards. This has a diameter of about $23 \mu \mathrm{m}$ whereas most other casts show a diameter of about $30 \mu \mathrm{m}$ (A). Casts contain remnants of tubular cells with condensed nuclear DNA (B).
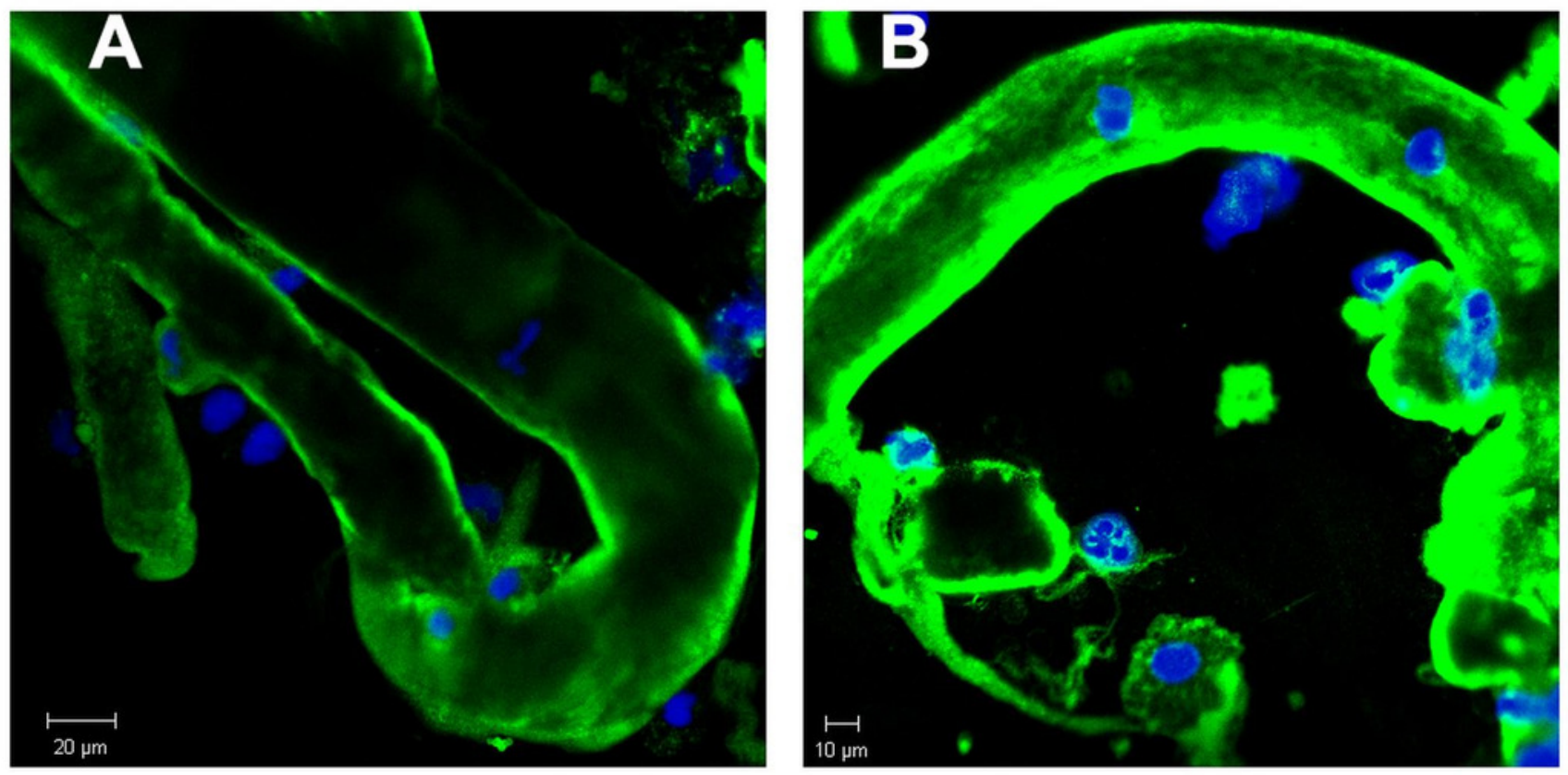


\section{Figure 3}

Sucrose density gradient separation of urine of patient 14 containing cast fragments, exosomes and micro-aggregates.

The centrifuged ( $40000 \mathrm{~g}$ for 2 hours) sucrose gradient (500 $\mu \mathrm{L}$ urine containing cast fragments and micro-aggregates) was fractionated into 14 individual fractions. Individual fractions were loaded onto a 12\% SDS PAGE gel, transferred to nitrocellulose and developed for lambda light chains (A). The same fractions were reprocessed and blotted for aquaporin 1 (AQP1) as a marker protein for heavy exosomes entering the dense sucrose layers such as in fractions 2-6 (B).
Sucrose 1.4
0.8
$0.3 \quad 0.25 \mathrm{M}$

$k D a$

25-

A

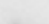

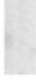

B

25-

$\begin{array}{lllllllllllllll}\text { Fractions } & 1 & 2 & 3 & 4 & 5 & 6 & 7 & 8 & 9 & 10 & 11 & 12 & 13 & 14\end{array}$ lambda

AQP1 


\section{Figure 4}

Urine lambda LC immunoblotting.

Twenty $\mu \mathrm{L}$ urine were loaded onto a $12 \%$ SDS PAGE gel and run under non-reducing conditions. The gel was blotted onto nitrocellulose and developed using a lambda LC-specific $\mathrm{Ab}$ (A). The clinical course of the patients and predominant LCs are given in Table $\mathbf{1}$. The same fractions were loaded onto a 12\% SDS PAGE gel and run under denaturing and reducing conditions. The gel was blotted onto nitrocellulose which was developed with the lambda LC-specific Ab. Only monomeric forms of LCs were depicted (B). Patients are numbered at the bottom of the picture in accordance to Table 1. The molecular weight is shown on the left side and light chain multimerization status is shown on the right. Ig stands for immunoglobulin. 


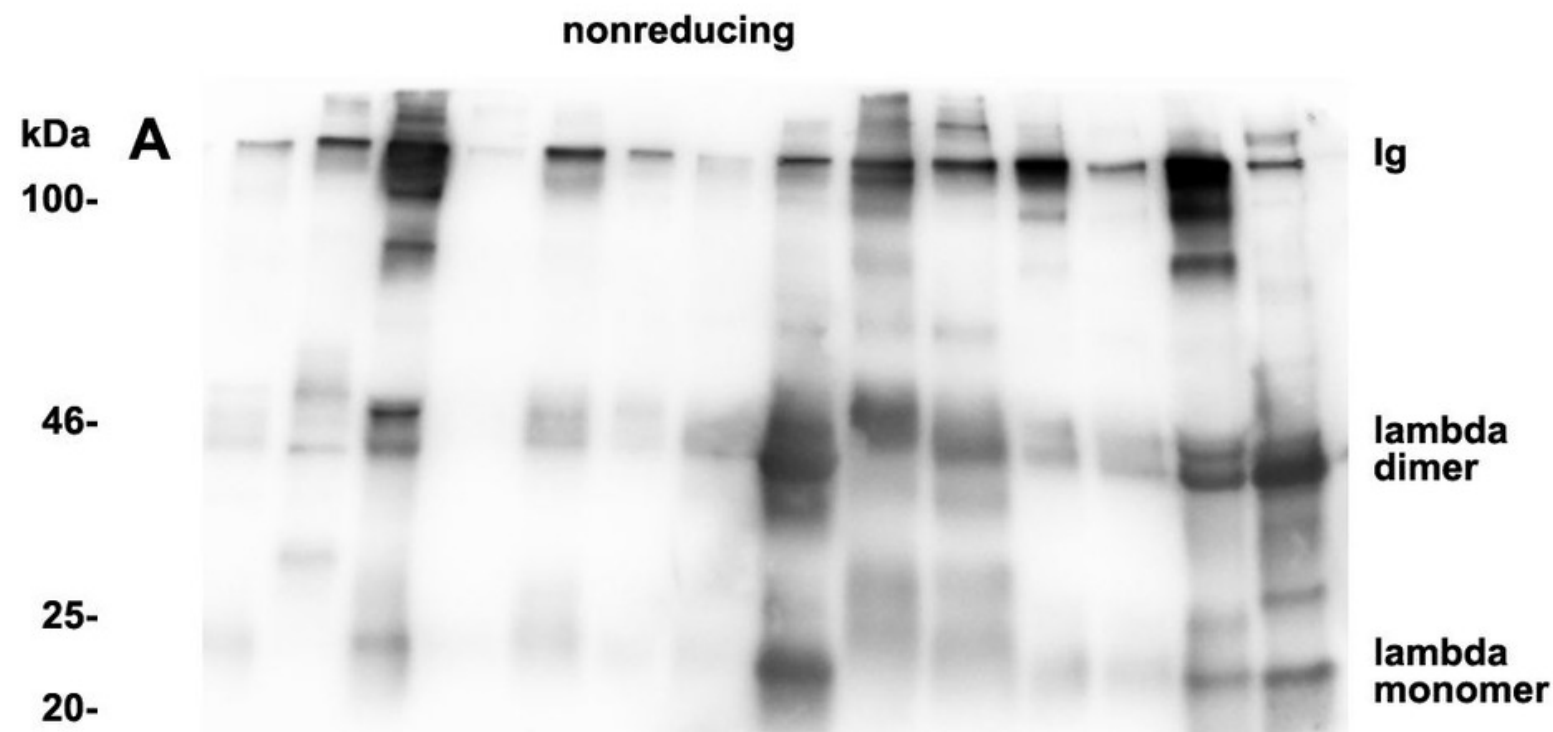

15-

10-

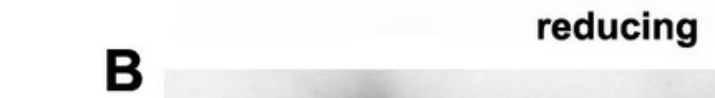

25-

Patients

$\begin{array}{llllllllllllll}1 & 2 & 3 & 4 & 5 & 6 & 7 & 8 & 9 & 10 & 11 & 12 & 13 & 14\end{array}$




\section{Figure 5}

Urine kappa LC immunoblotting

Twenty $\mu \mathrm{L}$ urine were loaded onto a 12\% SDS PAGE gel and run under non-reducing conditions. The gel was blotted onto nitrocellulose and developed using a kappa LC-specific $\mathrm{Ab}$ (A). The patients' clinical conditions and predominant LC are illustrated in Table 1. The same fractions were loaded onto a 12\% SDS PAGE gel and run under reducing and denaturing conditions. The gel was blotted onto nitrocellulose which was developed with the kappa LC-specific Ab. Only monomeric forms of LCs were depicted (B). Patients are numbered at the bottom of the picture in accordance to Table $\mathbf{1}$. The molecular weight is shown on the left side and light chain multimerization status is shown on the right. Ig stands for immunoglobulin. 


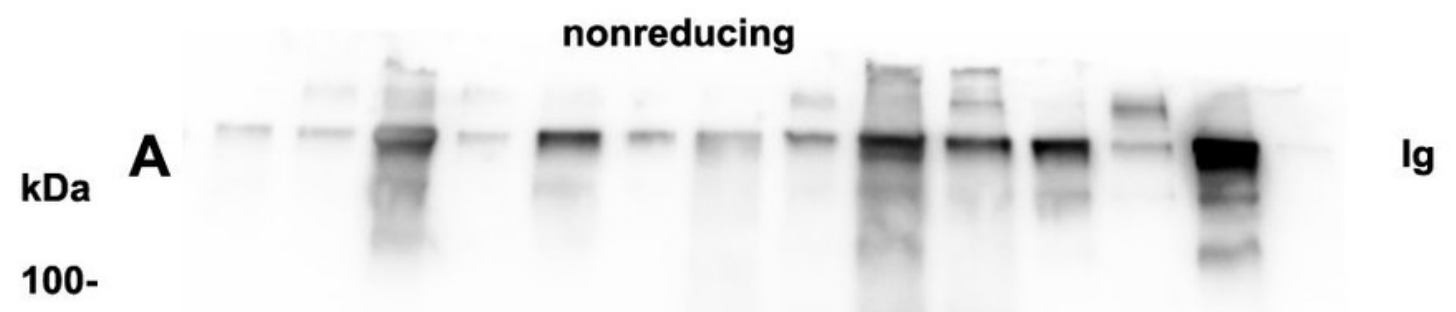

46-

kappa

dimer

25-
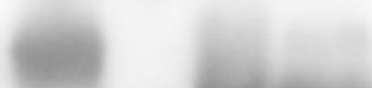

20-

15-

10-

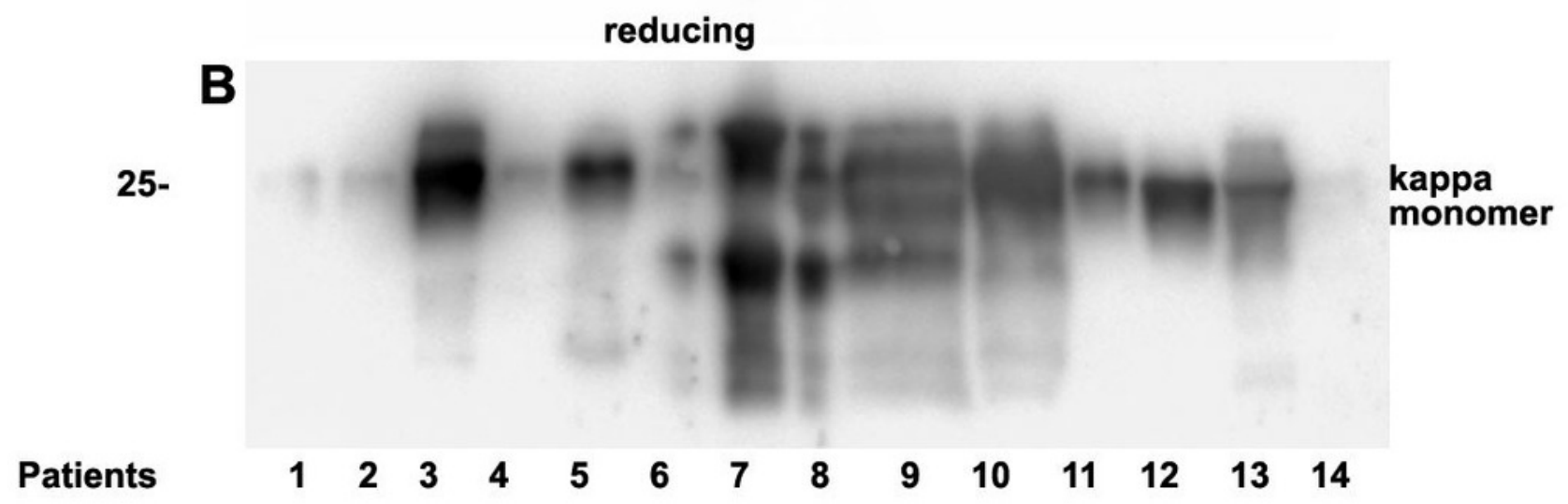




\section{Figure 6}

Sucrose density gradient separation of $500 \mu \mathrm{L}$ urine of patient 13 containing cast fragments, micro-aggregates and exosomes.

The centrifuged ( $40000 \mathrm{~g}$ for 2 hours) sucrose gradient was fractionated into 14 individual fractions which were loaded onto a 12\% SDS PAGE gel, transferred to nitrocellulose and developed for lambda light chains. Molecular weight is depicted on the left, lambda dimers and lambda monomers are indicated at the right, fraction numbering is indicated at the bottom and sucrose density at the top (A). The same fractions were tested for AQP1 (B) and for uromodulin concentration by ELISA (C). 


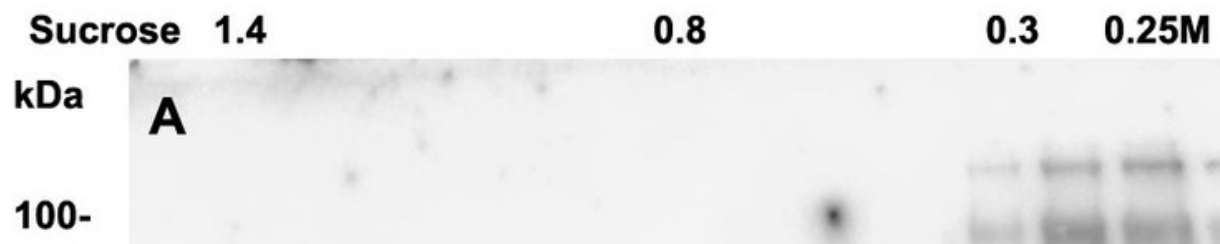

46-

lambda

dimer

25-

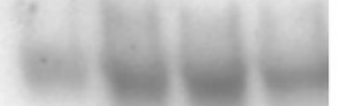

\section{lambda}

monomer

25-

AQP1

B

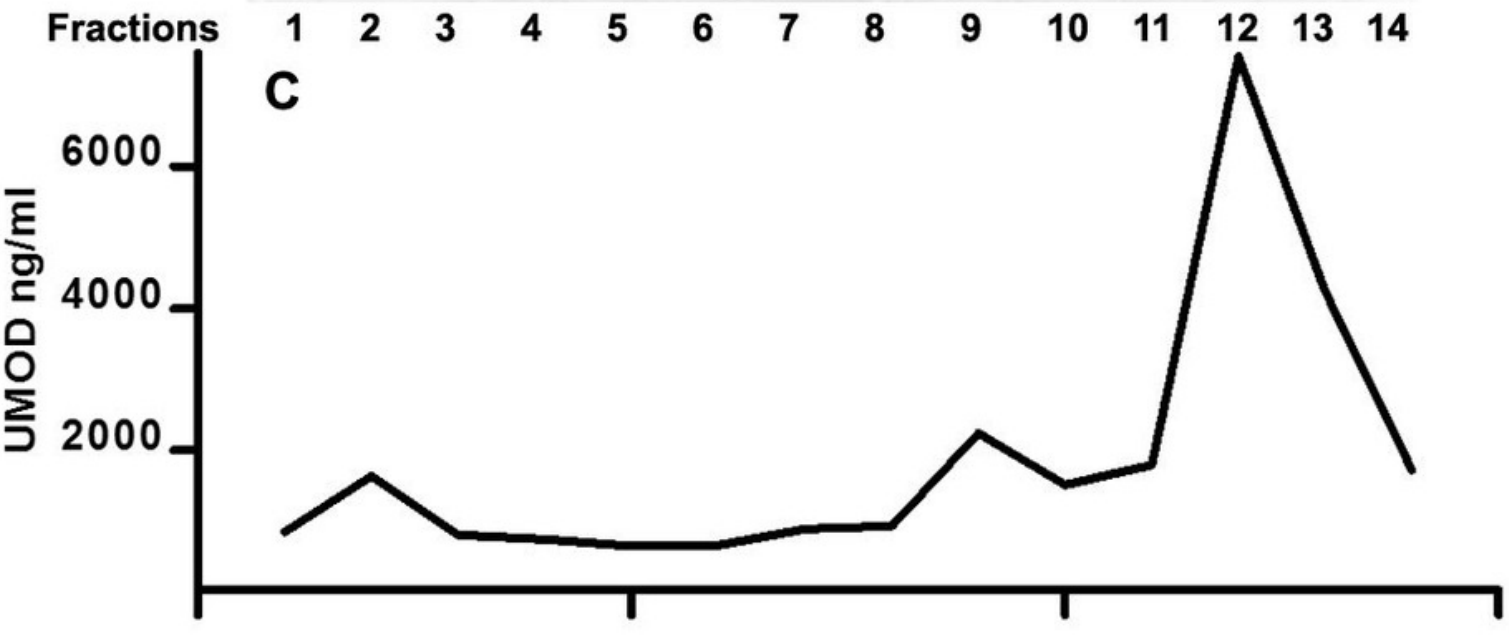

$\begin{array}{lllllllllllllll}\text { Fractions } & 1 & 2 & 3 & 4 & 5 & 6 & 7 & 8 & 9 & 10 & 11 & 12 & 13 & 14\end{array}$ 


\section{Figure 7}

Sucrose density gradient separation of urine containing cast fragments, microaggregates and exosomes from patient 15.

The centrifuged ( $40000 \mathrm{~g}$ for 2 hours) sucrose gradient (500 $\mu \mathrm{L}$ urine containing cast fragments and micro-aggregates) was fractionated into 14 individual fractions. Individual fractions were loaded onto a 12\% SDS PAGE gel, transferred to nitrocellulose and developed for kappa light chains. The legend to kappa monomers and dimers is indicated on the right (A). The same fractions were tested for AQP1 (B) and for UMOD by ELISA (C). 


$\begin{array}{lllll}\text { Sucrose } & 1.4 & 0.8 & 0.3 & 0.25 \mathrm{M} \\ \mathrm{kDa} & & & & \end{array}$

100 -

A

46-

kappa

dimer

25-

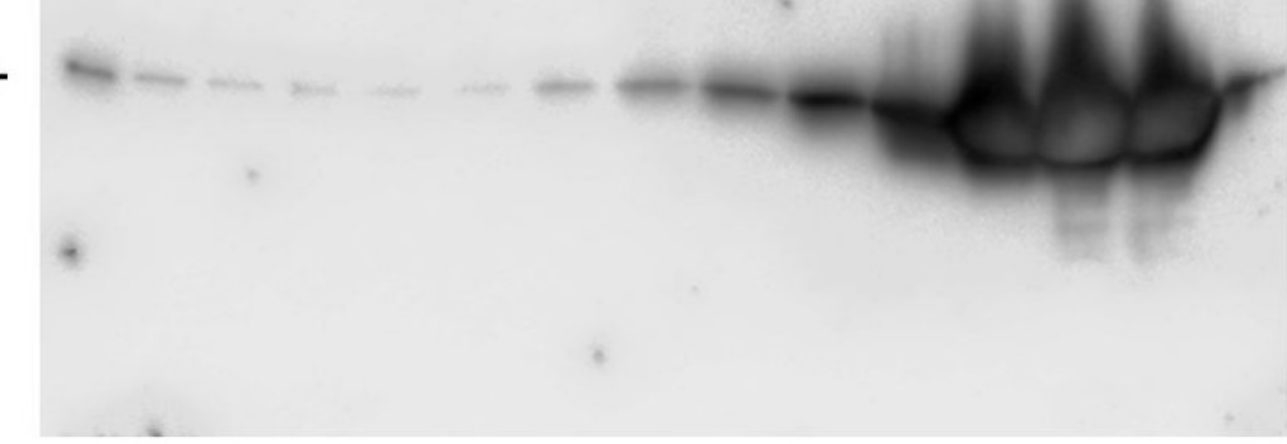

kappa

monomer

B

25-

AQP1

$\begin{array}{lllllllllllllll}\text { Fractions } & 1 & 2 & 3 & 4 & 5 & 6 & 7 & 8 & 9 & 10 & 11 & 12 & 13 & 14\end{array}$

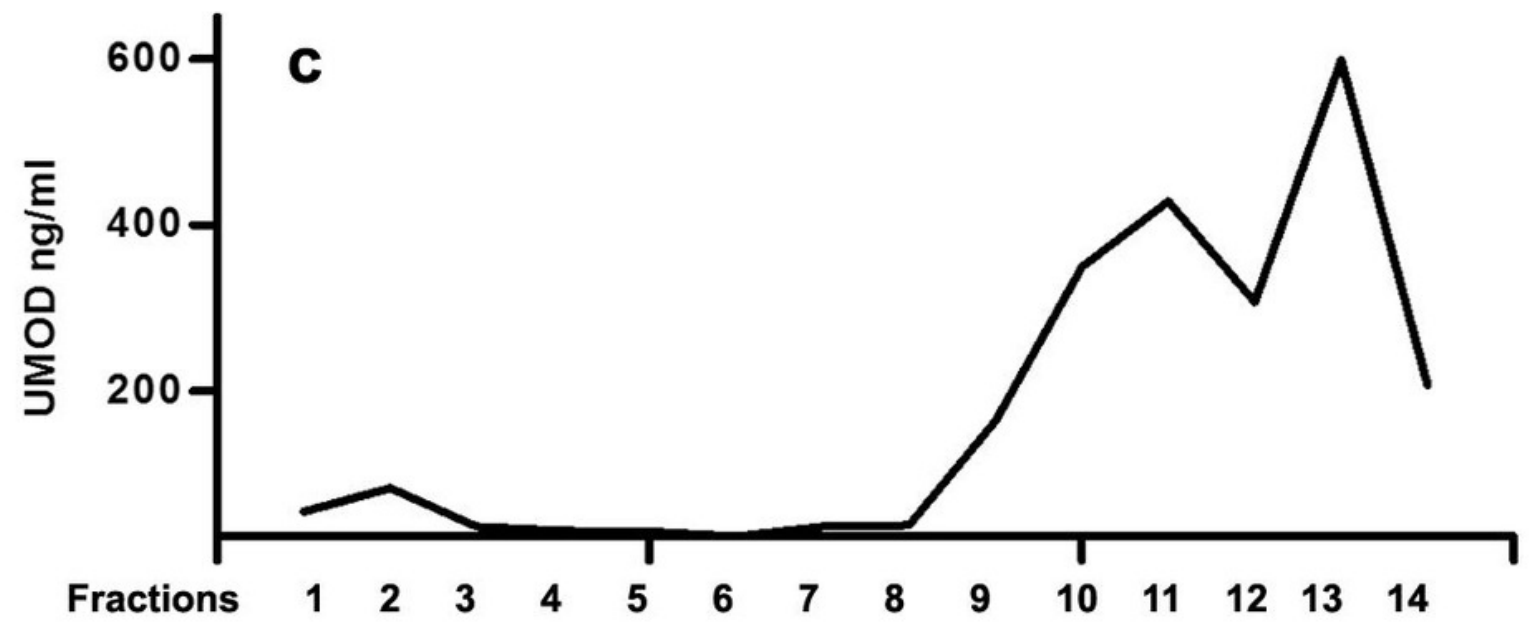




\section{Table $\mathbf{1}$ (on next page)}

Demographics, clinical characteristics and specification of light chain associated kidney disorder.

Abbreviations: AKI, acute kidney injury; CKD, chronic kidney disease; f, female; ID, identification; LC, light chain; LCCN, Light-chain cast nephropathy; LCPT, light-chain proximal tubulopathy; m, male; MGRS, monoclonal gammopathy of renal significance; MGUS, monoclonal gammopathy of undetermined significance; MM, multiple myeloma; nd, not done; ns, not shown; sCr, serum creatinine; pos, positive; TMA, thrombotic microangiopathy; U:P/C, urinary protein/creatinine ratio.

${ }^{\circ}$ extrarenal manifestation of amyloidosis, * at time of diagnosis.

Previous clinical therapy: 1: Bortezomib, 2: Thalidomide, 3: Cyclophosphamide, 4: Dexamethasone, 5: Carfilzomib, 6: Lenalidomid, 7: Daratumumab, 8: Rituximab. 


\begin{tabular}{|c|c|c|c|c|c|c|c|c|c|c|c|c|}
\hline ID & $\begin{array}{l}\text { Age } \\
\text { (years) }\end{array}$ & Gender & LC & $\begin{array}{l}\text { Hematological } \\
\text { Classification }\end{array}$ & $\begin{array}{l}\text { Renal } \\
\text { Histology }\end{array}$ & $\begin{array}{l}\text { Disease } \\
\text { Duration } \\
\text { (years) }\end{array}$ & $\begin{array}{l}\mathrm{sCr} * \\
(\mathrm{mg} / \mathrm{dL})\end{array}$ & $\begin{array}{l}\text { Urinary } \\
\text { Cast }\end{array}$ & CKD/AKI & $\begin{array}{l}\mathrm{U}: \mathrm{P} / \mathrm{C} \\
(\mathrm{mg} / \mathrm{g})\end{array}$ & $\begin{array}{l}\text { Previous } \\
\text { Therapy }\end{array}$ & Illustration \\
\hline 1 & 69 & $\mathrm{f}$ & $\lambda$ & $\mathrm{MM}$ & LCPT & 1 & 1.24 & 0 & 1 & 112 & 1,3 & Fig $4 \& 5 /$ lane 1 \\
\hline 2 & 76 & $\mathrm{f}$ & $\lambda$ & MM & nd & 10 & 0.81 & 0 & 1 & 81 & 5,6 & Fig $4 \& 5 /$ lane 2 \\
\hline 3 & 76 & $\mathrm{~m}$ & $\lambda$ & MGRS & LCPT & 4 & 1.2 & 0 & 4 & 1714 & & Fig $4 \& 5 /$ lane3 \\
\hline 4 & 87 & $\mathrm{f}$ & $\kappa$ & MM & nd & 6 & 1.07 & 0 & 2 & 1 & 8 & Fig $4 \& 5$ / lane 4 \\
\hline 5 & 63 & $\mathrm{f}$ & $\kappa$ & MM & nd & 6 & 0.61 & 0 & 1 & 274 & $1,3,4$ & Fig $4 \& 5 /$ lane 5 \\
\hline 6 & 49 & $\mathrm{~m}$ & $\kappa$ & $\mathrm{MM}^{\circ}$ & nd & 1 & 1.12 & 0 & 2 & 214 & $1,4,7$ & Fig $4 \& 5$ / lane 6 \\
\hline 7 & 75 & $\mathrm{~m}$ & $\kappa$ & MM & nd & 0 & 2.12 & 0 & 5 & 697 & 3 & Fig $4 \& 5$ / lane 7 \\
\hline 8 & 57 & $\mathrm{f}$ & $\lambda$ & $\mathrm{MM}^{\circ}$ & $\mathrm{LCCN}$ & 5 & 5.33 & 0 & 5 & 767 & $1,2,4$ & Fig $4 \& 5 /$ lane 8 \\
\hline 9 & 62 & $\mathrm{~m}$ & $\lambda$ & MGUS & nd & 0 & 0.84 & 0 & 4 & 476 & & Fig $4 \& 5$ / lane9 \\
\hline 10 & 63 & $\mathrm{~m}$ & $\lambda$ & MGUS & nd & 0 & 1.05 & 0 & 4 & 273 & & Fig $4 \& 5 /$ lane 10 \\
\hline 11 & 67 & $\mathrm{f}$ & $\lambda$ & $\mathrm{MM}^{\circ}$ & TMA & 4 & 2.02 & 0 & 4 & 222 & 1,4 & Fig $4 \& 5 /$ lane 11 \\
\hline 12 & 80 & $\mathrm{~m}$ & $\kappa$ & MM & nd & 0 & 1.04 & 0 & 2 & 126 & $2,4,5$ & Fig $4 \& 5 /$ lane 12 \\
\hline 13 & 67 & $\mathrm{f}$ & $\lambda$ & $\mathrm{MM}^{\circ}$ & $\begin{array}{l}\text { LCCN, } \\
\text { LCPT, AL- } \\
\text { amyloidosis }\end{array}$ & 1 & 1.59 & pos & 5 & 5631 & 1,7 & Fig $4 \& 5$ / lane13; Fig 6 \\
\hline 14 & 55 & $\mathrm{~m}$ & $\lambda$ & MM & $\mathrm{LCCN}$ & 5 & 1.22 & pos & $4 / 3$ & 4781 & 1 & Fig 4\&5 / lane14; Fig3; Sup. Fig 1 \\
\hline 15 & 59 & $\mathrm{~m}$ & $\kappa$ & MM & $\mathrm{LCCN}$ & 1 & 6.58 & pos & $3 / 3$ & 5561 & 1,2 & Fig 7 \\
\hline 16 & 75 & $\mathrm{~m}$ & $\lambda$ & $\mathrm{MM}^{\circ}$ & $\begin{array}{l}\mathrm{LCCN}, \mathrm{AL}- \\
\text { amyloidosis }\end{array}$ & 0 & 4.2 & pos & 5 & 4643 & & ns \\
\hline 17 & 48 & $\mathrm{~m}$ & $\lambda$ & $\mathrm{MM}^{\circ}$ & nd & 7 & 0.92 & 0 & 1 & 38 & $1,2,4$ & ns \\
\hline
\end{tabular}




\section{Table 2 (on next page)}

Proteome analysis using NanoLC-MS Analysis of 5 individual casts selected under microscopic observation by micromanipulation.

Five hyaline casts were picked from a cytoslide and analytically processed. Abundance of peptides is calculated as area under the curve (Norm. Area) of highly specific peptide peaks. Shown is one analysis out of three. 


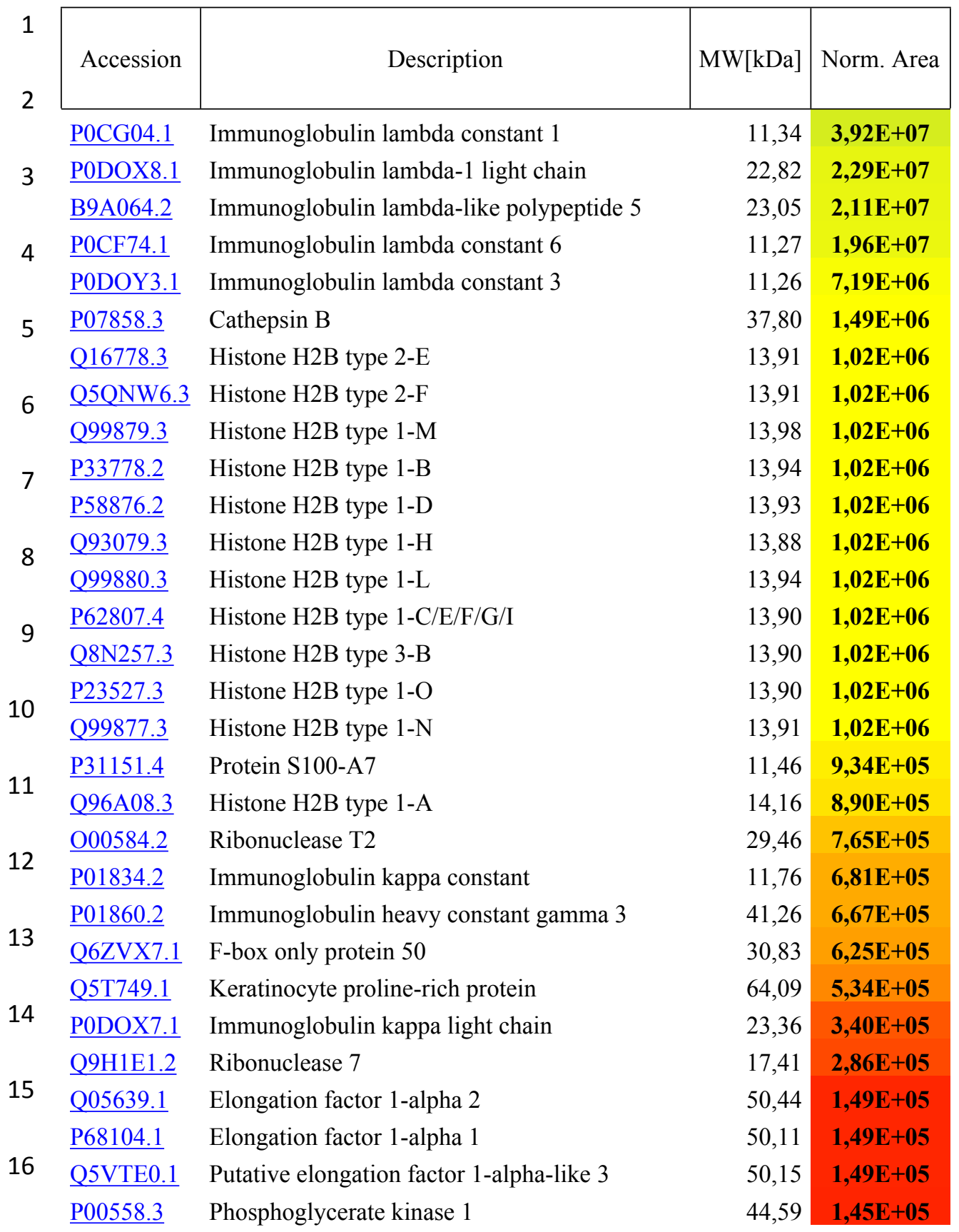

